\title{
On a Characteristic of Data Transmission with Priority Processing in Bus Network
}

\author{
M. Ooki
}

\begin{abstract}
Recently, in bus network each station is connected to a bus through a switching-hub which establishes a link from a sending station to a receiving station, so that the number of data collisions extremely decreased. However, in the case that the receiving buffer of a receiving station is full or the receiving station is sending or receiving data, a sending station has to wait to send data. This waiting time (stand-by time) influences an efficiency of data transmission. In this paper, a characteristic of data transmission with priority processing in bus network with the stand-by time is clarified.

Taking note of one station with two sending buffers for high priority data and low priority data, the link probability that the link from the station to a receiving station can be established is defined as a constant. The station is modeled as a priority queue with stand-by time, and then in steady state, a balance equation of the probabilities of the number of waiting data at the ready time of sending is expressed. From the equations, for each priority, probabilities of the number of waiting data mean of the number of waiting data in the sending buffer and mean waiting time which is the time from the data arrival time at the sending buffer to the beginning time of sending are derived. The calculated values are illustrated in several cases, and then the influences of the stand-by time and the link probability on data transmission with priority processing are shown.
\end{abstract}

Index Terms-Bus network, priority, link probability, mean of the number of waiting data, mean waiting time.

\section{INTRODUCTION}

In bus network which is a primary topology for LAN (local area network), recently, stations are connected to a bus through a switching hub which establishes a link from a sending station to a receiving station. By using a switching hub, the number of data collisions extremely decreased in comparison with the conventional protocol CSMA/CD (carrier sense multiple access / collision detection), so that a sending station did not need to almost wait to send data.

However, in this bus network with a switching hub, a sending station also has to wait for sending in the case that a receiving buffer at a receiving station is full or the receiving station is sending or receiving data.

As studies in conjunction with this paper, the proposal of MAC (media access control) protocol [1], the simulation of MAC protocol for the performance evaluation [2] and the proposal of the dynamic bandwidth allocation [3] for Ethernet PON (passive optical network) were presented. And, the time synchronization on $\mathrm{Gb} / \mathrm{s}$ Ethernet [4] which is necessary for

Manuscript received September 27, 2014; revised March 29, 2015.

M. Ooki is with the Dept. of Integrated System Engineering, Computer System Course, Nishinippon Institute of Technology, 1-11 Aratsu, Kanda-machi, Miyako-gun, Fukuoka, 800-0394 Japan (e-mail: ooki@nishitech.ac.jp). the wide distributed system, the host configuration mechanism to network in Ethernet and wireless LAN [5] are studied. For application of Ethernet, an application method in industry [6], application to production factory [7], the performance of industrial Ethernet [8] and the performance of TDMA (Time Division Multiple Access) based network [9] are reported. And, about the Adhoc network, impact of mobility [10], [11] and routing protocol [12], [13] are studied.

However, the characteristics of data transmission (probabilities of the number of waiting data, mean of the number of waiting data and mean waiting time) were not cleared, so that I reported simulations for a bus network with CSMA/CD [14], [15] and the characteristic of data transmission in bus network with switching hub [16].

In the case of transmission of different kinds of data, such as text, sound and graphics, priority processing is necessary. In this paper, a characteristic of data transmission with priority processing in bus network is analyzed.

In this paper, the probability that a link from a sending station to a receiving station can be established (called as link probability) is defined as a constant. Taking note of one station connected to a bus, for high priority data and low priority data, probabilities of the number of waiting data, mean of the number of waiting data in the sending buffer and mean waiting time from the arrival time at the sending buffer to the beginning time of sending [16]-[18] are derived. Furthermore, some calculated values are illustrated, and then characteristics of data transmission with priority processing are considered.

\section{Modeling OF Bus Network WITH PrIORITY PROCESSING}

The bus network shown in Fig. 1 is analyzed.

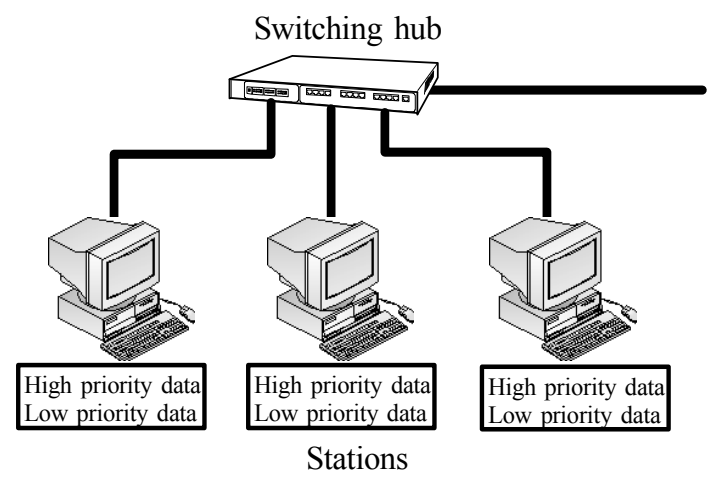

Fig. 1. Bus network with priority processing.

In the network, each station has two infinite buffers for high priority data and low priority data which are called as sending 
buffers. High priority data and low priority data arrive at each sending buffer in different Poisson process. The arrival rates of high priority data and low priority data are denoted by $\lambda_{1}$ and $\lambda_{2}$, respectively. At the ready time of sending data, if high priority data exist in the sending buffer and a link to a receiving station is established, the station sends the first high priority datum in the sending buffer to a receiving station. If not established, the station has a little waiting time (called as stand-by time). If there is no high priority data in the sending buffer, the station checks the sending buffer for low priority data. If low priority data exist in the sending buffer and a link to a receiving station is established, the station sends the first low priority datum in the sending buffer to the receiving station. If not established, the station has stand-by time. If there is no low priority data, the station has stand-by time. Defining the link probability as $q$, the flowchart of sending data is shown in Fig. 2.

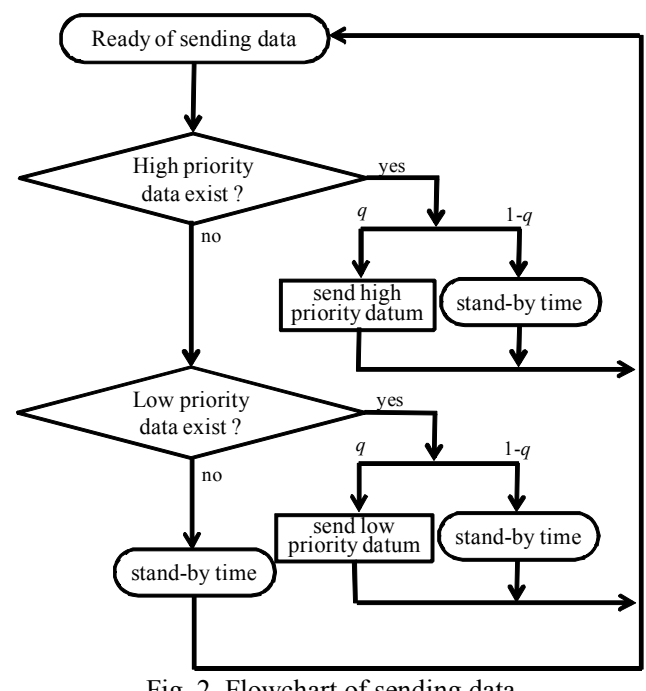

Fig. 2. Flowchart of sending data.

The sending station is modeled as a priority queue with stand-by time which is shown in Fig. 3.

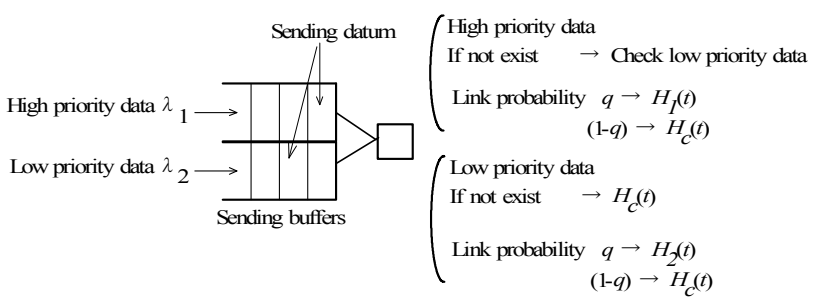

Fig. 3. Priority queue with stand-by time.

It is defined that one transmission requires arbitrary distributed transmission time. The distribution functions (DFs) of the transmission times for high priority data and low priority data are denoted by $H_{1}(t)$ and $H_{2}(t)$, respectively. The stand-by time is arbitrary distributed, and the DF of the stand-by time is denoted by $H_{c}(t)$.

\section{Probability of The Number of WAiting DATA AND MEAN OF THE NUMBER OF WAITING DATA}

\section{A. Balance Equation}

Let $P_{n_{1}, n_{2}}$ be the probability that the numbers of waiting high priority data and low priority data in the sending buffers are $n_{1}$ and $n_{2}$ at the ready time of sending. In steady state, the following balance equation is obtained.

$$
\begin{aligned}
& P_{n_{1}^{\prime}, n_{2}}=q \int_{0}^{\infty} \sum_{n_{1}=1}^{n_{1}^{\prime}+1} \alpha_{1}\left(n_{1}^{\prime}-n_{1}+1, t\right) \sum_{n_{2}=0}^{n_{2}{ }^{\prime}} \alpha_{2}\left(n_{2}{ }^{\prime}-n_{2}, t\right) P_{n_{1}, n_{2}} d H_{1}(t) \\
& +(1-q) \int_{0}^{\infty} \sum_{n_{1}=1}^{n_{1}{ }^{\prime}} \alpha_{1}\left(n_{1}^{\prime}-n_{1}, t\right) \sum_{n_{2}=0}^{n_{2}{ }^{\prime}} \alpha_{2}\left(n_{2}{ }^{\prime}-n_{2}, t\right) P_{n_{1}, n_{2}} d H_{c}(t) \\
& +q \int_{0}^{\infty} \alpha_{1}\left(n_{1}^{\prime}, t\right) \sum_{n_{2}=1}^{n_{2}+1} \alpha_{2}\left(n_{2}{ }^{\prime}-n_{2}+1, t\right) P_{0, n_{2}} d H_{2}(t) \\
& +(1-q) \int_{0}^{\infty} \alpha_{1}\left(n_{1}{ }^{\prime}, t\right) \sum_{n_{2}=1}^{n_{2}^{\prime}} \alpha_{2}\left(n_{2}{ }^{\prime}-n_{2}, t\right) P_{0, n_{2}} d H_{c}(t) \\
& +\int_{0}^{\infty} \alpha_{1}\left(n_{1}^{\prime}, t\right) \alpha_{2}\left(n_{2}{ }^{\prime}, t\right) P_{0,0} d H_{c}(t)
\end{aligned}
$$

In the above equation, $\alpha_{i}(n, t)$ is the probability that $n$ data arrive at the sending buffer within time $t$.

$$
\alpha_{i}(n, t)=\frac{e^{-\lambda_{i} t}\left(\lambda_{i} t\right)^{n}}{n !}
$$

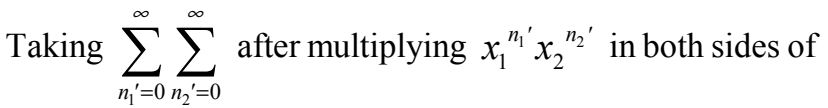
equation (1) and transforming into a generating function form, the following equation is obtained.

$$
G_{1}\left(x_{1}, x_{2}\right)=\frac{G_{2}\left(x_{2}\right)\left\{\frac{q}{x_{2}} H_{2}^{*}(z)+(1-q) H_{c}^{*}(z)-1\right\}+P_{0,0}\left\{H_{c}^{*}(z)-1\right\}}{1-\frac{q}{x_{1}} H_{1}^{*}(z)-(1-q) H_{c}^{*}(z)}
$$

where $H_{i}^{*}(z)$ and $H_{c}{ }^{*}(z)$ are the Laplace-Stieltjes Transformation(LST) of $H_{i}(t)$ and $H_{c}(t)$, respectively, and $z=\lambda_{1}\left(1-x_{1}\right)+\lambda_{2}\left(1-x_{2}\right)$.

The generating functions (GFs) of the probabilities of the number of waiting data are defined as follows.

$$
\begin{gathered}
G_{1}\left(x_{1}, x_{2}\right)=\sum_{n_{1}=1}^{\infty} \sum_{n_{2}=0}^{\infty} P_{n_{1}, n_{2}} x_{1}^{n_{1}} x_{2}^{n_{2}} \\
G_{2}\left(x_{2}\right)=\sum_{n_{2}=1}^{\infty} P_{0, n_{2}} x_{2}^{n_{2}}
\end{gathered}
$$

\section{B. Probabilities of the Number of Waiting Data}

When $x_{1}=x_{2}=1$ in equation (3), the following equations are obtained.

$$
\begin{aligned}
& \left\{q-q \lambda_{1} h_{1}-(1-q) \lambda_{1} h_{c}\right\} G_{1}(1,1)- \\
& \left\{q \lambda_{1} h_{2}+(1-q) \lambda_{1} h_{c}\right\} G_{2}(1)-\lambda_{1} h_{c} P_{0,0}=0
\end{aligned}
$$




$$
\begin{aligned}
& \left\{q \lambda_{2} h_{1}+(1-q) \lambda_{2} h_{c}\right\} G_{1}(1,1)- \\
& \left\{q-q \lambda_{2} h_{2}-(1-q) \lambda_{2} h_{c}\right\} G_{2}(1)+\lambda_{2} h_{c} P_{0,0}=0
\end{aligned}
$$

In equation (5a) and (5b), $h_{i}$ and $h_{c}$ are mean of $H_{i}(t)$ and $H_{c}(t)$, respectively.

Because the sum of all probabilities is 1, equation (6) is obtained.

$$
G_{1}(1,1)+G_{2}(1)+P_{0,0}=1
$$

From equations (5a), (5b) and (6), the following probabilities are derived.

$$
\begin{gathered}
G_{1}(1,1)=\frac{\lambda_{1} h_{c}}{q\left\{1-\left(\lambda_{1} h_{1}+\lambda_{2} h_{2}\right)+\left(\lambda_{1}+\lambda_{2}\right) h_{c}\right\}} \\
G_{2}(1)=\frac{\lambda_{2} h_{c}}{q\left\{1-\left(\lambda_{1} h_{1}+\lambda_{2} h_{2}\right)+\left(\lambda_{1}+\lambda_{2}\right) h_{c}\right\}} \\
P_{0,0}=\frac{q\left\{1-\lambda_{1} h_{1}-\lambda_{2} h_{2}+\left(\lambda_{1}+\lambda_{2}\right) h_{c}\right\}-\left(\lambda_{1}+\lambda_{2}\right) h_{c}}{q\left\{1-\left(\lambda_{1} h_{1}+\lambda_{2} h_{2}\right)+\left(\lambda_{1}+\lambda_{2}\right) h_{c}\right\}}
\end{gathered}
$$

$G_{1}(1,1)$ is the probability that the number of high priority waiting data is 1 or more at the ready time of sending. $G_{2}(1)$ is the probability that the number of high priority waiting data is 0 and low priority data is 1 or more. $P_{0,0}$ is the probability that there is no waiting data.

\section{Mean of the Number of High Priority Waiting Data}

From equation (3), $\left.\frac{d}{d x_{1}} G_{1}\left(x_{1}, 1\right)\right|_{x_{1}=1}$ which is mean of the number of high priority waiting data in the sending buffer can be derived.

From equation (3), $G_{1}\left(x_{1}, 1\right)$ is expressed by equations (8), (9a) and (9b).

$$
\begin{gathered}
G_{1}\left(x_{1}, 1\right)=\frac{A_{1}\left(x_{1}\right)}{B_{1}\left(x_{1}\right)} \\
A_{1}\left(x_{1}\right)=G_{2}(1)\left\{q H_{2}^{*}\left(z_{1}\right)+(1-q) H_{c}^{*}\left(z_{1}\right)-1\right\} \\
+P_{0,0}\left\{H_{c}^{*}\left(z_{1}\right)-1\right\} \\
B_{1}\left(x_{1}\right)=1-\frac{q}{x_{1}} H_{1}^{*}\left(z_{1}\right)-(1-q) H_{c}^{*}\left(z_{1}\right)
\end{gathered}
$$

where $z_{1}=\lambda_{1}\left(1-x_{1}\right)$.

When $x_{1}=1$ after differentiating equation (8), the following equation is obtained

$$
\left.\frac{d}{d x_{1}} G_{1}\left(x_{1}, 1\right)\right|_{x_{1}=1}=\frac{A_{1}^{\prime \prime}(1) B_{1}{ }^{\prime}(1)-A_{1}{ }^{\prime}(1) B_{1}{ }^{\prime \prime}(1)}{2\left\{B_{1}{ }^{\prime}(1)\right\}^{2}}
$$

where

$$
\begin{gathered}
A_{1}^{\prime}(1)=G_{2}(1)\left\{q \lambda_{1} h_{2}+(1-q) \lambda_{1} h_{c}\right\}+P_{0,0} \lambda_{1} h_{c} \\
B_{1}^{\prime}(1)=q-q \lambda_{1} h_{1}-(1-q) \lambda_{1} h_{c}
\end{gathered}
$$

and

$$
A_{1}^{\prime \prime}(1)=G_{2}(1)\left\{q \lambda_{1}^{2} h_{2}^{(2)}+(1-q) \lambda_{1}^{2} h_{c}^{(2)}\right\}+P_{0,0} \lambda_{1}^{2} h_{c}^{(2)}
$$

$$
B_{1}^{\prime \prime}(1)=-2 q+2 q \lambda_{1} h_{1}-q \lambda_{1}^{2} h_{1}^{(2)}-(1-q) \lambda_{1}^{2} h_{c}^{(2)}
$$

$h_{i}^{(2)}$ and $h_{c}^{(2)}$ are the second moments of the DFs of $H_{i}(t)$ and $H_{c}(t)$, respectively.

\section{Mean of the Number of Low Priority Waiting Data}

Using $x_{1}=\delta\left(x_{2}\right)$ which satisfies that the denominator of equation (3) is 0 , the following equation is obtained.

$$
G_{2}\left(x_{2}\right)=\frac{P_{0,0}\left\{H_{c}^{*}(\tilde{z})-1\right\}}{1-\frac{q}{x_{2}} H_{2}^{*}(\tilde{z})+(1-q) H_{c}^{*}(\tilde{z})}
$$

where $\tilde{z}=\lambda_{1}\left\{1-\delta\left(x_{2}\right)\right\}+\lambda_{2}\left(1-x_{2}\right)$ and $\delta\left(x_{2}\right)$ satisfies the following equation.

$$
1-\frac{q}{\delta\left(x_{2}\right)} H_{1}^{*}(\tilde{z})-(1-q) H_{c}^{*}(\tilde{z})=0
$$

From equation (13), $\left.\frac{d}{d x_{2}} G_{2}\left(x_{2}\right)\right|_{x_{2}=1}$ which is mean of the number of low priority waiting data in the case that the number of high priority waiting data is 0 can be derived. From equation (13), $G_{2}\left(x_{2}\right)$ is expressed by equations (15), (16a) and (16b).

$$
\begin{gathered}
G_{2}\left(x_{2}\right)=\frac{A_{2}\left(x_{2}\right)}{B_{2}\left(x_{2}\right)} \\
A_{2}\left(x_{2}\right)=P_{0,0}\left\{H_{c}^{*}(\tilde{z})-1\right\} \\
B_{2}\left(x_{2}\right)=1-\frac{q}{x_{2}} H_{2}^{*}(\tilde{z})-(1-q) H_{c}^{*}(\tilde{z})
\end{gathered}
$$

When $x_{2}=1$ after differentiating equation (15), the following equation is obtained.

$$
\left.\frac{d}{d x_{2}} G_{2}\left(x_{2}\right)\right|_{x_{2}=1}=\frac{A_{2}^{\prime \prime}(1) B_{2}^{\prime}(1)-A_{2}^{\prime}(1) B_{2}^{\prime \prime}(1)}{2\left\{B_{2}^{\prime}(1)\right\}^{2}}
$$




$$
\begin{gathered}
A_{2}^{\prime}(1)=P_{0,0}\left(-h_{c}\right) \tilde{z}_{0}^{\prime} \\
B_{2}^{\prime}(1)=q-q\left(-h_{2}\right) \tilde{z}_{0}^{\prime}-(1-q)\left(-h_{c}\right) \tilde{z}_{0}^{\prime}
\end{gathered}
$$

where $\tilde{z}_{0}^{\prime}=\left.\tilde{z}^{\prime}\right|_{x_{2}=1}$, and

$$
\begin{aligned}
& A_{2}^{\prime \prime}(1)=P_{0,0} h_{c}^{(2)}\left(\tilde{z}_{0}^{\prime}\right)^{2}+P_{0,0}\left(-h_{c}\right)\left(\tilde{z}_{0}^{\prime \prime}\right)^{2} \\
& B_{2}^{\prime \prime}(1)=-2 q+2 q\left(-h_{2}\right) \tilde{z}_{0}^{\prime}-q h_{2}^{(2)}\left(\tilde{z}_{0}^{\prime}\right)^{2}-q\left(-h_{2}\right) \tilde{z}_{0}^{\prime \prime} \\
& -(1-q) h_{c}^{(2)}\left(\tilde{z}_{0}^{\prime}\right)^{2}-(1-q)\left(-h_{c}\right) \tilde{z}_{0}^{\prime \prime}
\end{aligned}
$$

where $\tilde{z}_{0}^{\prime \prime}=\left.\tilde{z}^{\prime \prime}\right|_{x_{2}=1}$.

On the other hand, from $\tilde{z}=\lambda_{1}\left\{1-\delta\left(x_{2}\right)\right\}+\lambda_{2}\left(1-x_{2}\right)$ and equation (14), the following equations are obtained.

$$
\begin{aligned}
& \tilde{z}_{0^{\prime}}=-\lambda_{1} \delta_{0}{ }^{\prime}-\lambda_{2} \\
& \delta_{0}{ }^{\prime}=\frac{q \lambda_{2} h_{1}+(1-q) \lambda_{2} h_{c}}{q-q \lambda_{1} h_{1}-(1-q) \lambda_{1} h_{c}} \\
& \tilde{z}_{0}{ }^{\prime \prime}=-\lambda_{1} \delta_{0}^{\prime \prime} \\
& \delta_{0}^{\prime \prime}=\frac{2 q\left(\delta_{0}^{\prime}\right)^{2}+2 q \delta_{0}^{\prime} h_{1} \widetilde{z_{0}^{\prime}}+q h_{1}^{(2)}\left(\widetilde{z_{0}^{\prime}}\right)^{2}+(1-q) h_{c}^{(2)}\left(\widetilde{z_{0}^{\prime}}\right)^{2}}{q-q \lambda_{1} h_{1}-(1-q) \lambda_{1} h_{c}}
\end{aligned}
$$

From these equations, $\tilde{z}_{0}{ }^{\prime}$ and $\tilde{z}_{0}{ }^{\prime \prime}$ can be calculated.

\section{Mean Waiting Time}

\section{A. High Priority}

The probability that the number of high priority waiting data at the end of sending data is $n_{1}^{\prime}$ is denoted by $R_{1 n_{1}^{\prime}}$, and then the following equation is obtained.

$$
R_{1 n_{1}^{\prime}}=\frac{1}{G_{1}(1,1)} \int_{0}^{\infty} \sum_{n_{1}=1}^{n_{1}{ }^{\prime}+1} \alpha_{1}\left(n_{1}^{\prime}-n_{1}+1, t\right) \sum_{n_{2}=0}^{\infty} P_{n_{1}, n_{2}} d H_{1}(t)
$$

Taking $\sum_{n_{1}{ }^{\prime}=0}^{\infty}$ after multiplying $x_{1}^{n_{1}{ }^{\prime}}$ in both sides in equation (21), and transforming into equation of the GF, equation (22) is obtained.

$$
G_{R_{1}}\left(x_{1}\right)=\frac{1}{x_{1} G_{1}(1,1)} G_{1}\left(x_{1}, 1\right) H_{1}^{*}\left(z_{1}\right)
$$

where $G_{R_{1}}\left(x_{1}\right)=\sum_{n_{1}^{\prime}=0}^{\infty} R_{1 n_{1}^{\prime}} x_{1}^{n_{1}^{\prime}}$.
On the other hand, the probability that the number of high priority arrival data during waiting time and sending time for high priority data is $n_{1}^{\prime}$ is equal to $R_{1 n_{1}{ }^{\prime}}$, so that the following equation is obtained.

$$
R_{1 n^{\prime}}=\int_{0}^{\infty} \alpha_{1}\left(n_{1}{ }^{\prime}, t\right) d W_{l}(t) \otimes H_{l}(t)
$$

where $W_{1}(t)$ is the DF of waiting time for high priority data and $\otimes$ is a convolution operator.

Taking $\sum_{n_{1}=0}^{\infty}$ after multiplying $x_{1}^{n_{1}^{\prime}}$ in both sides of equation (23) and transforming into equation of the GF, equation (24) is derived.

$$
G_{R 1}\left(x_{1}\right)=W_{1}^{*}\left(z_{1}\right) H_{1}^{*}\left(z_{1}\right)
$$

where $W_{1}^{*}\left(z_{1}\right)$ is the LST of $W_{1}(t)$.

From equation (22) and (24), $W_{1}^{*}(z)$ is obtained as follows.

$$
W_{1}^{*}\left(z_{1}\right)=\frac{1}{x_{1} G_{1}(1,1)} G_{1}\left(x_{1}, 1\right)
$$

When $z_{1}=0$ after differentiating equation (25) by $z_{1}$, the left side of equation is obtained as follows.

$$
\left.\frac{d W_{1}^{*}\left(z_{1}\right)}{d z_{1}}\right|_{z_{1}=0}=-w_{1}
$$

where $w_{1}$ is mean of $W_{1}(t)$.

On the other hand, the right side is obtained as follows.

$$
\begin{aligned}
& \left.\frac{d}{d z_{1}} \frac{G_{1}\left(x_{1}, 1\right)}{x_{1} G_{1}(1,1)}\right|_{z_{1}=0}=\left.\frac{d}{d x_{1}} \frac{G_{1}\left(x_{1}, 1\right)}{x_{1} G_{1}(1,1)} \frac{d x_{1}}{d z_{1}}\right|_{x_{1}=1} \\
& =-\left.\frac{1}{\lambda_{1}} \frac{d}{d x_{1}} \frac{G_{1}\left(x_{1}, 1\right)}{x_{1} G_{1}(1,1)}\right|_{x_{1}=1} \\
& =-\left.\frac{1}{\lambda_{1} G_{1}(1,1)} \frac{\frac{d}{d x_{1}} G_{1}\left(x_{1}, 1\right) x_{1}-G_{1}\left(x_{1}, 1\right)}{x_{1}^{2}}\right|_{x_{1}=1} \\
& =-\frac{1}{\lambda_{1} G_{1}(1,1)}\left\{\left.\frac{d}{d x_{1}} G_{1}\left(x_{1}, 1\right)\right|_{x_{1}=1}-G_{1}(1,1)\right\}
\end{aligned}
$$

From equation (26a) and equation (26b), mean waiting time for high priority data $w_{1}$ is derived.

$$
w_{1}=\frac{1}{\lambda_{1} G_{1}(1,1)}\left\{\left.\frac{d}{d x_{1}} G_{1}\left(x_{1}, 1\right)\right|_{x_{1}=1}-G_{1}(1,1)\right\}
$$


$G_{1}(1,1)$ and $\left.\frac{d}{d x_{1}} G_{1}\left(x_{1}, 1\right)\right|_{x_{1}=1}$ have been already derived, so that mean waiting time $w_{1}$ can be calculated.

\section{B. Low Priority}

Similar to the analysis for high priority, the following two equations are obtained.

$$
\begin{gathered}
R_{2 n_{2}{ }^{\prime}}=\frac{1}{G_{2}(1)} \int_{0}^{\infty} \sum_{n_{2}=1}^{n_{2}{ }^{+}+1} \alpha_{2}\left(n_{2}{ }^{\prime}-n_{2}+1, t\right) P_{0, n_{2}} d H_{2}(t) \\
R_{2 n_{2}{ }^{\prime}}=\int_{0}^{\infty} \alpha_{2}\left(n_{2}{ }^{\prime}, t\right) d W_{2}(t) \otimes H_{2}(t)
\end{gathered}
$$

where $R_{2 n_{2}{ }^{\prime}}$ is the probability that the number of low priority waiting data at the end of sending data is $n_{2}{ }^{\prime}$ and $W_{2}(t)$ is the DF of waiting time for low priority data.

Taking $\sum_{n_{2}{ }^{\prime}=0}^{\infty}$ after multiplying $x_{2}^{n_{2}^{\prime}}$ in both sides in equations (28a) and (28b), and transforming into equations of the GF, equation (29) is derived.

$$
W_{2}^{*}\left(z_{2}\right)=\frac{1}{x_{2} G_{2}(1)} G_{2}\left(x_{2}\right)
$$

where $W_{2}^{*}\left(z_{2}\right)$ is the LST of $W_{2}(t)$.

From equation (29), mean waiting time for low priority $w_{2}$ is derived as follows.

$$
w_{2}=\frac{1}{\lambda_{2} G_{2}(1)}\left\{\left.\frac{d}{d x_{2}} G_{2}\left(x_{2}\right)\right|_{x_{2}=1}-G_{2}(1)\right\}
$$

$$
G_{2}(1) \text { and }\left.\frac{d}{d x_{2}} G_{2}\left(x_{2}\right)\right|_{x_{2}=1} \text { have been already derived, so }
$$

that mean waiting time $w_{2}$ can be calculated.

\section{Numerical Examples}

Fig. 4 shows a relation between arrival ratio $\lambda_{1}$ and mean waiting time $w_{1}$ and $w_{2}$ in the case of $\lambda_{1}=\lambda_{2}, q=0.1,0.5$, $0.9, h_{1}=h_{2}=5, h_{c}=1$ and $H(t)$ and $H_{c}(t)$ obey uniform distributions.

In Fig. 4, solid lines, dashed lines and chain lines are mean waiting times in the case of $q=0.1,0.5$ and 0.9 , respectively. In the same line, the lower line is mean waiting time $w_{1}$ and the upper line is mean waiting time $w_{2}$. It is confirmed that the mean waiting time becomes long as the link probability $q$ becomes low and as the arrival ratio $\lambda$ becomes high. In the case of $q=0.1$, mean waiting time $w_{1}$ and $w_{2}$ become infinite when $\lambda_{1}$ is over 0.0685 and 0.0335 , respectively.
This means that when $q=0.1$ and $\lambda_{1}$ is over 0.0685 and 0.0335 , each sending buffer is overflow because there are more arrival data than sending data.

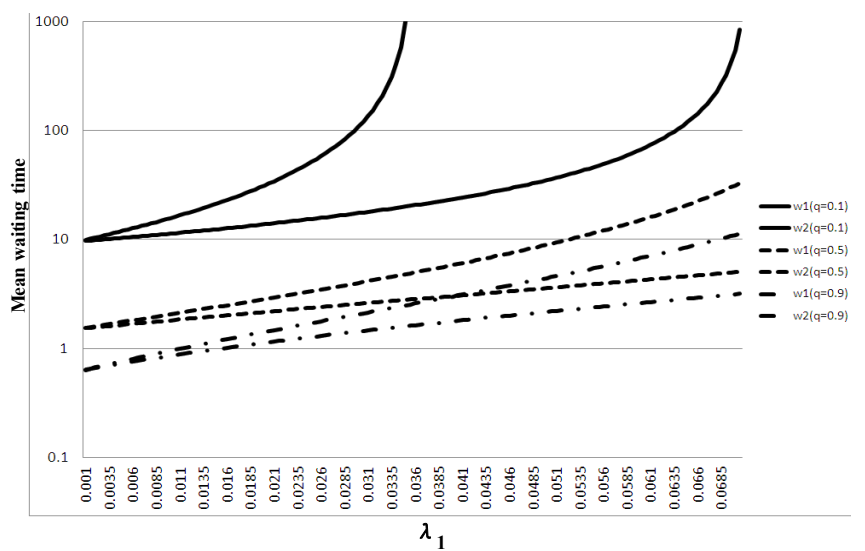

Fig. 4. Mean waiting time $w_{1}$ and $w_{2}\left(h_{1}=h_{2}=5, h_{c}=1, \lambda_{1}=\lambda_{2}\right.$, uniform distribution).

In the case that $H(t)$ and $H_{c}(t)$ obey exponential distributions, Fig. 5 is illustrated.

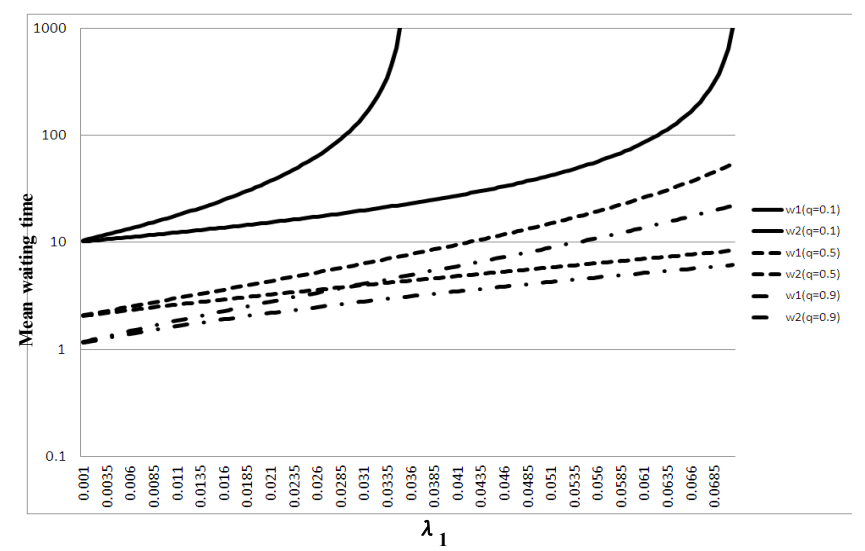

Fig. 5. Mean waiting time $w_{1}$ and $w_{2}\left(h_{1}=h_{2}=5, h_{c}=1, \lambda_{1}=\lambda_{2}\right.$, exponential distribution).

Comparing Fig. 5 with Fig. 4, the mean waiting times become long, but Fig. 5 shows similar tendency.

Next, in the case of $q=0.5, \lambda_{1}=\lambda_{2}$ and $\lambda_{2}=3 \lambda_{1}$, the mean waiting times are shown in Fig. 6.

Mean waiting times in the case of $\lambda_{2}=3 \lambda_{1}$ become longer than those in the case of $\lambda_{1}=\lambda_{2}$. Particularly, mean waiting time $w_{2}$ becomes very long. And, mean waiting time $w_{1}$ slightly becomes long in spite of same $\lambda_{1}$. If an arrival rate of low priority data rises, it is natural that waiting time for low priority data becomes longer, but that for high priority data slightly becomes long. This reason is that the opportunities when low priority data are sent increase. But, there is little influence on the waiting time for high priority data.

Mean waiting times in the case of $\lambda_{2}=\lambda_{1} / 3$ become shorter than those in the case of $\lambda_{1}=\lambda_{2}$. Particularly, mean waiting time $w_{1}$ slightly becomes short in spite of same $\lambda_{1}$.

From Fig. 6 and Fig. 7, it is confirmed that mean waiting time for high priority is slightly influenced by the arrival ratio of low priority data. 


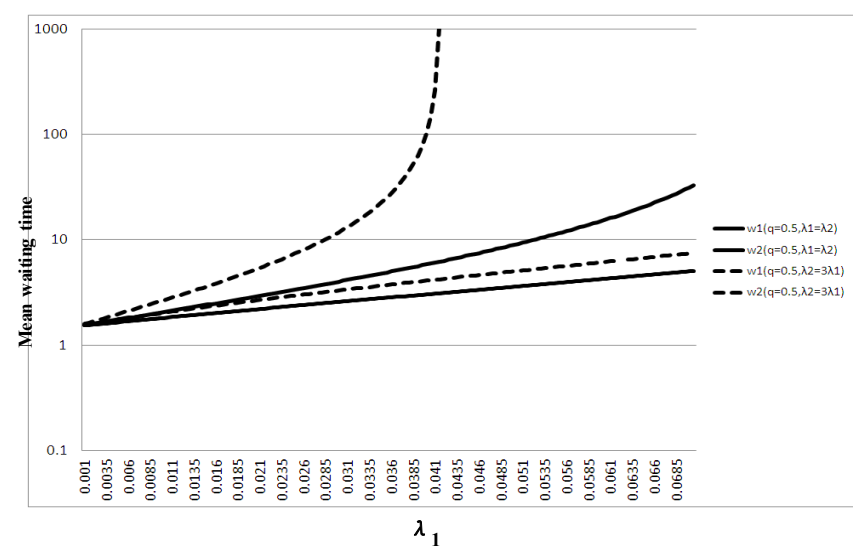

Fig. 6. Mean waiting time $w_{1}$ and $w_{2}\left(h_{1}=h_{2}=5, h_{c}=1, \lambda_{1}=\lambda_{2}\right.$, and $\lambda_{2}=3 \lambda_{1}$ uniform distribution).

Fig. 7 shows mean waiting times in the case of $q=0.5$, $\lambda_{1}=\lambda_{2}$ and $\lambda_{2}=\lambda_{1} / 3$.

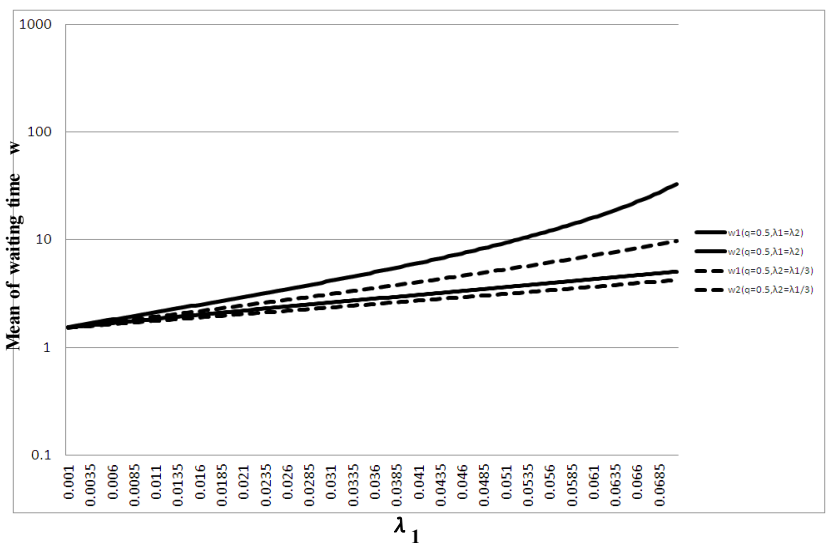

Fig. 7. Mean waiting time $w_{1}$ and $w_{2}\left(h_{1}=h_{2}=5, h_{c}=1, \lambda_{1}=\lambda_{2}\right.$ and $\lambda_{2}=\lambda_{1} / 3$, uniform distribution).

\section{CONCLUSION}

In this paper, a characteristic of data transmission with priority processing in bus network with switching hub is clarified. In the analysis, taking note of one station connected to the bus, the link probability that the link from the station to a receiving station can be established is defined as a constant, and then for each priority data, the probabilities of the number of waiting data, the mean of the number of waiting data at the ready time of sending and the mean waiting times are derived. For the analysis of low priority, a root that the denominator of GF is equal to 0 is used.

Furthermore, some numerical examples are illustrated and the influence of the link probability and the ratio of $\lambda_{1}$ and $\lambda_{2}$ on the characteristics of data transmission are clarified.

The characteristic of data transmission with priority processing in this paper is a base of considering efficiency of bus network with priority processing. And, the analysis method in this paper may be applied to other networks.

The following considerations remain for further study:

1) Analyzing the bus network with more priority levels.

2) Clarifying a relation between the link probability and the buffer size of a receiving station.

\section{REFERENCES}

[1] M. Ma, Y. Zhu, and T. H. Cheng, "Bandwidth guaranteed polling MAC protocol for Ethernet passive optical networks," in Proc. IEEE INFOCOM, 2003, no. 1, pp. 22-31.

[2] J. Eom, Y. Chang, and S. H. Kim, "Bandwidth allocation and performance analysis of MAC protocol for Ethernet PON," J. Inst. Electron Eng. Korea TC, vol. 40, no. 7, pp. 261-272, 2003.

[3] Y. Luo and N. Ansari, "Dynamic upstream bandwidth allocation over Ethernet PONs," in Proc. IEEE Int. Conf. on Communication, 2005, no. 3, pp. 1853-1857.

[4] Y. Yamada, S. Ohta, and H. Uematsu, "Hardware-based precise time synchronization on $\mathrm{Gb} / \mathrm{s}$ Ethernet enhanced with preemptive priority," IEICE Trans. on Commun., vol. E89-B, no. 3, pp. 683-689, 2006.

[5] T. Huang and K. Chu, "Networking without dynamic host configuration protocol server in Ethernet and wireless local area network," J. Netw. Comput. Appl., vol. 34, no. 6, pp. 2027-2041, 2011.

[6] J. Decotignie, "Ethernet-based real-time and industrial communications," Proceedings of IEEE, vol. 93, no. 6, pp. 1102-1117.

[7] J. Putter, "Multiprotocol Ethernets on the shop-floor," in Information Infrastructure Systems for Manufacturing, J. Goossenaerts, F. Kimura, and H. Wortmann, Eds. 1997, pp. 335-343.

[8] J. Jaspernette, M. Schumacher, and K. Weber, "Limits of increasing the performance of industrial protocols," in Proc. IEEE Int. Conf. Emerge. Tecnol. Fact. Autom., 2007, vol. 12, pp. 17-24.

[9] N. Otsuki and T. Sugiyama, "Implementation of a TDMA based wireless network coding prototype system with Ethernet frame aggregation," IEICE Trans. on Commun., vol. E95-B, no. 12, pp. 3752-3759, 2012.

[10] F. Bay, N. Sadagopan, and A. Helmy, "Important: A framework to systematically analyze the impact of mobility on performance of routing protocol for Ad-hoc networks," in Proc. IEEE INFOCOM, 2003, no. 2, pp. 825-835.

[11] T. Hara, "Quantifying impact of mobility on data availability in mobile Ad-hoc networks," IEEE Trans. Mob. Comput., vol. 9, no. 2, pp. 241-258, 2010.

[12] K. Hasebe et al., "Muliple-route Ad-hoc on Demand (MRAODV) routing protocol," Report of IPSJ, no. 21, pp. 1-6, 2003.

[13] S. Rao, B. Mcdonald, and F. Znati, "Design and analysis of hierarchical group-based mobility for Ad-hoc wireless networks," in Proc. Communication Network Distribution System Model Simul. Conf., 2001, pp. 26-33.

[14] R. Zhao and M. Ooki, "Simulation for an Ethernet with two stations," Memories of Nishinippon Institute of Technology, vol. 36, pp. 19-24, 2006.

[15] M. Ooki, "On a transmission characteristic of Ethernet," Memories of Nishinippon Institute of Technology, vol. 40, pp. 21-26, 2010.

[16] M. Ooki, "On a characteristic of data transmission in bus network," $J$. of Advances in Computer Networks, vol. 2, no. 2, pp. 120-124, 2014.

[17] M. Ooki, Y. Fukagawa, S. Murakami, and S. Yoshida, "An approximate analysis of an asymmetric multiqueue with nonpreemptive priority and cyclic service discipline (in Japanese)," Transactions of the Institute of Electronics and Communication Engineers, vol. J72-A, no. 10, pp. 1563-1573, 1989.

[18] M. Ooki, Y. Fukagawa, S. Murakami, and S. Yoshida, "Analysis of nonpreemptive priority queues with service disciplines depending on the number of waiting messages," Asia-Pacific Journal of Operational Research, vol. 10, pp. 57-78, 1993.

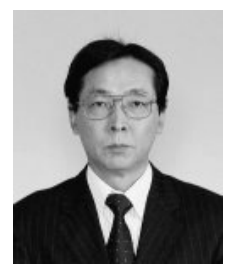

M. Ooki was born in Fukuoka, Japan on January 27, 1953. He got the doctor degree engineering form Kyushu Institute of Technology Graduate School in 1992. His major field is communication network system.

$\mathrm{He}$ is a professor of Nishinippon Institute of Technology, Fukuoka, Japan.

Dr. Ooki is a member of Electronics, Information and Communication Engineers Society, Information Processing Society and Operations Research Society in Japan. 